\title{
Reviewers for 2017
}

The Ghana Medical Journal appreciates the contribution of individuals who support its peer- review process. Our reviewers work quietly but effectively to maintain the standards and the quality of the manuscripts that finally get published. The Ghana Medical Journal is grateful for the voluntary service provided by the following reviewers for the year 2017:

\begin{tabular}{|c|c|c|c|c|c|}
\hline Abang, Innocent & Nigeria & Darko, Rudolph & Ghana & Shittu, Akeem & Nigeria \\
\hline Abdulai, Alhassan & Ghana & Darko, Rudolph & Ghana & Spangenberg, Kathryn & Ghana \\
\hline Aboagye, Anthony & Ghana & Dei-Adomakoh, Yvonne & Ghana & Tachi, Kenneth & Ghana \\
\hline Abrahams, Afua & Ghana & Dogbe, Edith & Ghana & Tette, Edem & Ghana \\
\hline Adabayeri, Victoria May & Ghana & Edwin, Frank & Ghana & Ukachukwu, Alvan & Nigeria \\
\hline Adeboye, Muhammed & Nigeria & Ekem, Ivy & Ghana & Vuvor, Frederick & Ghana \\
\hline Adei, Eunice & Ghana & Enimil, Anthony & Ghana & Wiredu, Edwin & Ghana \\
\hline Adeniran, Abiodun & Nigeria & \multicolumn{2}{|c|}{ Enweronu-Laryea, Christabel Ghana } & Yawson, Alfred & Ghana \\
\hline Adeyemi, Adewale & Nigeria & Esimai, Peju & Nigeria & Yeboah-Manu, Dorothy & Ghana \\
\hline Adisa, Rasaq & Nigeria & Essuman, Akye & Ghana & Yorke, Ernest & Ghana $\bullet$ \\
\hline Adusei, Ben & Ghana & Faried, Kyei & Ghana & & \\
\hline Adzamli, Innocent & Ghana & Fiscian, Henrietta & Ghana & & \\
\hline Afaa, Taiba & Ghana & Forson, Audrey & Ghana & & \\
\hline Agbenorku, Pius & Ghana & Gumanga, Solomon & Ghana & & \\
\hline Agyekum, Francis & Ghana & Isara, Alphonsus & Nigeria & & \\
\hline Ahorlu, Collins & Ghana & Johnson, Ofonime & Nigeria & & \\
\hline Aikins, Moses & Ghana & Kitcher, Emmanuel & Ghana & & \\
\hline Akaba, Godwin & Nigeria & Koram, Kwadwo & Ghana & & \\
\hline Akhigbe, Kingsley & Nigeria & Kyei, Mathew & Ghana & & \\
\hline Akpalu, Albert & Ghana & Larsen-Reindorf, Rita & Ghana & & \\
\hline Akpalu, Josephine & Ghana & Lartey, Margaret & Ghana & & \\
\hline Amoako, Yaw & Ghana & Lartey, Seth & Ghana & & \\
\hline Amoaku, Winfried & UK & Lasisi, Akeem & Nigeria & & \\
\hline \multicolumn{2}{|c|}{ Amoyaw-Asamoah, Isabella Ghana } & Lawson, Henry & Ghana & & \\
\hline Andrews, Nii Bonney & Ghana & Malm, Keziah & Ghana & & \\
\hline Anim, J Tei & Ghana & Mante, Sunny & Ghana & & \\
\hline Ankobiah, William & Ghana & Mensah, samuel & Ghana & & \\
\hline Antwi-Agyei, K. Odei & Ghana & Mensah, Yaw & Ghana & & \\
\hline Antwi, Sampson & Ghana & Morhe, Emmanuel & Ghana & & \\
\hline Anyanechi, Charles & Nigeria & Ndanu, Thomas & Ghana & & \\
\hline Appiah-Opong, Regina & Ghana & Newman, Mercy & Ghana & & \\
\hline Archampong, Timothy & Ghana & Nimako-Boateng, Kwasi & Ghana & & \\
\hline Asuquo, Marcus & Nigeria & Nkyekyer, Kobina & Ghana & & \\
\hline Awuku, Mark & Canada & Nwafor, Chukwuemeka & Nigeria & & \\
\hline Ayinmode, Adekunle & Nigeria & Oboirien, Muhammad & Nigeria & & \\
\hline Badoe, Ebenezer & Ghana & Ofori-Adjei, David & Ghana & & \\
\hline Badu-Peprah, Augustina & Ghana & Ofori-Adjei, Yaw & Ghana & & \\
\hline Baidoo, Kenneth & Ghana & Ohene, Sally-Ann & Ghana & & \\
\hline \multicolumn{2}{|c|}{ Bediako-Bowan, Antoinette Ghana } & Olowofela, Abimbola & Nigeria & & \\
\hline Biritwum, Richard & Ghana & Onyeonoro, Ugochukwu & Nigeria & & \\
\hline Boima, Vincent & Ghana & Osafo, Charlotte & Ghana & & \\
\hline Bonney, Agatha & Ghana & Osei-Tutu, Benjamin & Ghana & & \\
\hline Bonney, Joseph & Ghana & Osunde, Otasowie & Nigeria & & \\
\hline Bonsu, Frank & Ghana & Puplampu, Peter & Ghana & & \\
\hline Braimah, Ramat & Nigeria & Renner, Lorna & Ghana & & \\
\hline Chinawa, Ndubuisi & Nigeria & Sackey, Adziri & Ghana & & \\
\hline da-Costa Vroom, Frances & Ghana & Seneadza, Nana & Ghana & & \\
\hline
\end{tabular}

